\title{
Reconstructing subsets of reals
}

\author{
A.J. Radcliffe ${ }^{1}$ \\ Department of Mathematics and Statistics \\ University of Nebraska-Lincoln \\ Lincoln, NE 68588-0323 \\ jradclif@math.unl.edu \\ A.D. Scott \\ Department of Mathematics \\ University College \\ Gower Street \\ London WC1E 6BT \\ A.Scott@ucl.ac.uk
}

Submitted: November 24, 1998; Accepted: March 15, 1999

${ }^{1}$ Partially supported by NSF Grant DMS-9401351 


\begin{abstract}
We consider the problem of reconstructing a set of real numbers up to translation from the multiset of its subsets of fixed size, given up to translation. This is impossible in general: for instance almost all subsets of $\mathbb{Z}$ contain infinitely many translates of every finite subset of $\mathbb{Z}$. We therefore restrict our attention to subsets of $\mathbb{R}$ which are locally finite; those which contain only finitely many translates of any given finite set of size at least 2 .

We prove that every locally finite subset of $\mathbb{R}$ is reconstructible from the multiset of its 3-subsets, given up to translation.
\end{abstract}

Primary: 05E99; Secondary: 05C60 


\section{Introduction.}

Reconstructing combinatorial objects from information about their subobjects is a long-standing problem. The Reconstruction Conjecture and the Edge Reconstruction Conjecture both deal with the problem of reconstructing a graph from a multiset of subgraphs; in one case the collection of all induced subgraphs with one fewer vertex, in the other the collection of all subgraphs with one fewer edge (see Bondy [2] and Bondy and Hemminger $[3])$.

The very general problem is that of reconstructing a combinatorial object (up to isomorphism) from the collection of isomorphism classes of its subobjects. Isomorphism plays a crucial rôle. Thus it seems that the natural ingredients for a reconstruction problem are a group action (to provide a notion of isomorphism) and an idea of what constitutes a subobject. Reconstruction problems have been considered from this perspective by, for instance, Alon, Caro, Krasikov and Roditty [1], Radcliffe and Scott [11], [10], Cameron [4], [5], and Mnukhin [7], [8], [9].

In this paper we consider the problem of reconstructing subsets of the groups $\mathbb{Z}, \mathbb{Q}$, and $\mathbb{R}$ from the multiset of isomorphism classes of their subsets of fixed size, where two subsets are isomorphic if one subset is a translate of the other. Where the subsets have size $k$ we call this collection the $k$-deck.

Maybe the first thing to notice is that for $|A| \geq k$ one can reconstruct the $l$-deck of $A$ from the $k$-deck for any $l \leq k$. This is a straightforward translation of Kelly's lemma (see [2]). On the other hand if $|A|<k$ then the $k$-deck of $A$ is empty, and therefore $A$ cannot be distinguished from any other subset of size strictly less than $k$. It makes the statement of our theorems slightly easier if we use a definition of deck for which this issue does not arise. The definition we adopt below regards the deck as a function on multisets of size $k$. It is straightforward to check that this form of the $k$-deck can be determined from the deck as defined above, provided $|A| \geq k$.

Definition 1 Let $A$ be a subset of $\mathbb{F}$, where $\mathbb{F}$ is one of $\mathbb{Z}, \mathbb{Q}$, or $\mathbb{R}$. The $k$-deck of $A$ is the function defined on multisets $Y$ of size $k$ from $\mathbb{F}$ by

$$
d_{A, k}(Y)=|\{i \in \mathbb{F}: \operatorname{supp}(Y+i) \subset A\}|,
$$

where $\operatorname{supp}(Y)$ is the set of elements of $Y$, considered without multiplicity. We say that $A$ is reconstructible from its $k$-deck if we can deduce $A$ up to 
THE ELECTRONIC JOURNAL of COMBinAtorics 6 (1999), \#R20

translation from its $k$-deck; in other words, we have

$$
d_{B, k} \equiv d_{A, k} \Rightarrow B=A+i, \text { for some } i \in \mathbb{F} \text {. }
$$

More generally we say that a function of $A$ is reconstructible from the $k$-deck of $A$ if its value can be determined from $d_{A, k}$.

Certain subtleties arise since the groups involved are infinite. It may be that the $k$-deck of $A \subset \mathbb{F}$ takes the value $\infty$ on some finite (multi)sets. In fact, for any fixed finite subset $F \subset \mathbb{Z}$, almost all subsets of $\mathbb{Z}$ (with respect to the obvious symmetric probability measure on $\mathcal{P}(\mathbb{Z})$ ) contain infinitely many translates of $F$. Thus it is trivial to find, for all $k \geq 1$, two subsets of $\mathbb{Z}$ with the same $k$-deck which are not translates of one another.

For this reason we restrict our attention to subsets $A \subset \mathbb{F}$ for which the 2-deck (and a fortiori the $k$-deck for all $k \geq 2$ ) takes only finite values, or equivalently, every distance occurs at most fintely many times. We shall call such sets locally finite.

It is easily seen that every finite subset $A \subset \mathbb{R}$ can be reconstructed from its 3 -deck, $d_{A, 3}$ : indeed, let $n=\operatorname{diam} A:=\max A-\min A$; then

$$
A \simeq\{0, n\} \cup\left\{r: d_{A, 3}(\{0, r, n\})>0\right\} .
$$

The 2-deck is not, however, in general enough. For instance, if $A$ and $B$ are finite sets of reals then $A+B$ and $A-B$ have the same 2-deck.

Our aim in this note is to prove a reconstruction result for locally finite sets of reals. We begin by proving a result for $\mathbb{Z}$ and work in stages towards $\mathbb{R}$. We shall write $A \simeq B$ if $A$ is a translation of $B$.

Theorem 1 Let $A \subset \mathbb{Z}$ be locally finite. Then $A$ is reconstructible from its 3-deck. In other words, if $A, B \subset \mathbb{Z}$ have the same 3-deck then $A \simeq B$.

We shall first prove a lemma. For subsets $A, B \subset \mathbb{Z}$, we define $A+B$ to be the multiset of all $a+b$ with $a \in A$ and $b \in B$. (This multiset might of course take infinte values). Thus, for finite $A$ and $B$, if we identify $A$ with $a(x)=\sum_{i \in A} x^{i}$ and $B$ with $b(x)=\sum_{i \in B} x^{i}$, then $A+B$ can be identified with $a(x) b(x)$, where the coefficient of $x^{i}$ in $a(x) b(x)$ is the multiplicity of $i$ in the sum $A+B$.

If $L$ is a multiset of $\mathbb{Z}$ we write $m_{L}(i)$ for the multiplicity of $i$ in $L$.

Lemma 2 Let $A, B, C \subset \mathbb{Z}$ be finite and suppose that $A+C=B+C$. Then $A=B$. 
Proof. Straightforward by induction on $|A|$, noting that $\min (A+C)=$ $\min A+\min C$.

Lemma 3 If $A, B \subset \mathbb{Z}$ are locally finite, infinite sets with $A \triangle B$ finite, and $C$ is a finite set with $A+C=B+C$ then $A=B$.

Proof. Let $A_{0}=A \backslash B$, let $B_{0}=B \backslash A$, and set $R=A \cap B$. Now for all $i$ we have

$$
\begin{aligned}
m_{A_{0}+C}(i) & =m_{A+C}(i)-m_{R+C}(i) \\
& =m_{B+C}(i)-m_{R+C}(i) \\
& =m_{B_{0}+C}(i) .
\end{aligned}
$$

Thus $A_{0}+C=B_{0}+C$ and it follows from Lemma 2 that $A_{0}=B_{0}$ and so $A=B$.

Lemma 4 If $A, B \subset \mathbb{Z}$ are locally finite, infinite sets, and $C$ is a finite set with $A+C=B+C$ then $A=B$.

Proof. We may suppose, without loss of generality, that $0 \in C$. Now let $S=\{i: C+i \subset A+C\}$ and $c=\operatorname{diam}(C)$. We aim to show that, except for a finite amount of confusion, we have $S=A$. To this end, let $N$ be sufficiently large such that for all distinct $a, a^{\prime} \in A$ with $|a|>N$ we have $\left|a^{\prime}-a\right|>4 c$ and for all distinct $b, b^{\prime} \in B$ with $|b|>N$ we have $\left|b^{\prime}-b\right|>4 c$. (Such an $N$ exists since $A$ and $B$ are locally finite.) Suppose now that $k$, with $|k|>N+4 c$, belongs to two sets from $\{C+i: i \in S\}$, say $k \in(C+i) \cap(C+j)$. Define $D=(C+i) \cup(C+j)$. Since $\operatorname{diam}(D)>c$, while $D \subset A+C$, there must be distinct elements $a_{1}, a_{2} \in A$ such that $D$ meets both $C+a_{1}$ and $C+a_{2}$. But this is impossible, for then $\left|a_{1}-a_{2}\right| \leq 4 c$, while $\left|a_{1}\right|>N$. Thus every $k \in A+C$ with $|k|>N+4 c$ belongs to exactly one set $C+i$. It follows that $i \in A$, and by the same reasoning $i \in B$.

Now set $R=\{i \in S:|i|>N+4 c\}$. We have just established that $R \subset A$ and $R \subset B$, and obviously $R \supset\{a \in A:|a|>N+4 c\}$ and $R \supset$ $\{b \in B:|b|>N+4 c\}$. Thus $A \Delta B$ is finite, and by Lemma 3 the result is established.

Lemma 5 Let $A, B \subset \mathbb{Z}$ be locally finite infinite sets and let $C, D \subset \mathbb{Z}$ be finite. If $A+C=B+D$ then $A \simeq B$ and $C \simeq D$. 
Proof. We may clearly assume that $\min C=\min D=0$. Under this hypothesis we will prove that $A=B$ and $C=D$.

We will show that $C$ (and equally $D$ ) is the largest set such that infinitely many translates of $C$ are contained in $A+C=B+D$. Suppose then that $A+C$ contains infinitely many translates of some set $E$ and that no translate of $E$ is a subset of $C$. Let $E_{1}, E_{2}, \ldots$ be translates of $E$, where $E_{i} \subset A+C$ and $\left|\min E_{i}\right| \rightarrow \infty$ as $i \rightarrow \infty$. Since $E$ is contained in no translate of $C$, every $E_{i}$ must meet at least two translates of $C$, say $C_{a_{i}}$ and $C_{b_{i}}$, where $a_{i}$ and $b_{i}$ are distinct elements of $A$. Thus there are distinct $a_{i}, b_{i} \in A$ with

$$
\left|a_{i}-b_{i}\right| \leq 2 \operatorname{diam}(C)+\operatorname{diam}(E)
$$

and $\left|a_{i}\right| \rightarrow \infty$; since there are only finitely many possibilities for $a_{i}-b_{i}$ and infinitely many $a_{i}$, some distance must occur infinitely many times, which contradicts the assumption that $A$ is locally finite.

We conclude that $C$ is the largest set (uniquely defined up to translation) that has infinitely many translates as subsets of $A+C$. Hence we have $C \simeq D$ and so $C \equiv D$, since $\min C=\min D$. Thus $A+C=B+D=B+C$, and by Lemma $4, A=B$.

Proof. [of Theorem 1] If $A$ is finite then it is easily reconstructed from its 3 -deck, as noted above. Thus we may assume that $A$ is infinite.

Let $k$ be a difference that occurs in $A$ (i.e. there are $a_{1}, a_{2} \in A$ with $\left.a_{1}-a_{2}=k\right)$. We shall show that $A$ can be reconstructed from its 3 -deck; moreover, it can be reconstructed from its 3 -deck restricted to multisets of the form $\{0, k, \alpha\}$. Indeed, let $B$ be another set with the same 3-deck. Define

$$
X_{A}=\{a \in A: a+k \in A\}
$$

and

$$
X_{B}=\{b \in B: b+k \in B\} .
$$

Then, translating if necessary, we may assume that $\min X_{A}=\min X_{B}$. We claim now that $A=B$.

In order to prove our result it is enough to show that $-A+X_{A}=-B+X_{B}$, for then the result follows immediately from Lemma 5: since $-A=-B$ we also have $A=B$.

Now for $i \in \mathbb{Z}$, the multiplicity of $i$ in $-A+X_{A}$ is

$$
\begin{aligned}
\mid\left\{j: j \in X_{A}, i-j \in-A\right\} & =\left|\left\{j: j \in X_{A}, j-i \in A\right\}\right| \\
& =|\{j: j, j+k, j-i \in A\}| .
\end{aligned}
$$


If $i \neq 0,-k$, then this is the multiplicity of $\{0, i, i+k\}$ in the 3-deck of $A$; if $i=0$ or $i=-k$ then this is $\left|X_{A}\right|$, the multiplicity of $\{0, k\}$ in the 2-deck of $A$. Clearly, similar calculations hold for $B$, so $-A+X_{A}=-B+X_{B}$.

Theorem 6 Lemmas 2, 3, 4, and 5 hold in $\mathbb{Z}^{n}$ for all positive integers $n$. Moreover if $A, B \subset \mathbb{Z}^{n}$ have the same 3-deck then $A \simeq B$.

Proof. The proofs are almost identical to those for the corresponding results about $\mathbb{Z}$. We use the norm $|a|=\|a\|_{2}$, and order $\mathbb{Z}^{n}$ lexicographically, so $a \leq b$ if the first nonzero coordinate of $b-a$ is positive. The assumptions $\min C=\min D$ in the proof of Lemma 2 and $\min X_{A}=\min X_{B}$ in the proof of Theorem 1 then make sense. Moreover, the claim in the proof of Lemma 4 that $\operatorname{diam}(D)>\operatorname{diam}(C)$ is easily seen to hold in $\mathbb{Z}^{n}$ also: suppose $D=(C+i) \cup(C+j)$ and $x, y \in C$ satisfy $|x-y|=\operatorname{diam}(C)$. Let $v=i-j \neq 0$. Now $|(x+i)-(y+j)|=|(x-y)+v|$ and $|(x+j)-(y+i)|=|(x-y)-v|$ and one of these two norms is strictly greater than $|x-y|=\operatorname{diam}(C)$ (by the strict convexity of the norm we have chosen).

Theorem 7 Let $A, B \subset \mathbb{Q}$ be locally finite and have the same 3-deck, then $A \simeq B$.

Proof. Suppose $A$ and $B$ are locally finite subsets of $\mathbb{Q}$ with the same 3deck. Let $k$ be some distance that occurs in $A$, and again define $X_{A}=$ $\{a \in A: a+k \in A\}$ and $X_{B}=\{b \in B: b+k \in B\}$ as in the proof of Theorem 1. We may assume $\min X_{A}=\min X_{B}=0$. Now suppose $n$ is an integer such that $1 / n$ divides $k$ and all differences in $X_{A}$ and $X_{B}$. That is, $n k \in \mathbb{Z}$ and for all $q, r \in X_{A} \cup X_{B}$ we have $n(q-r) \in \mathbb{Z}$. In particular $n q \in \mathbb{Z}$ for all $q \in X_{A} \cup X_{B}$. We will show that for all $i$ we have

$$
A \cap \frac{1}{i n} \mathbb{Z}=B \cap \frac{1}{i n} \mathbb{Z}
$$

Since $\mathbb{Q}=\bigcup_{i \geq 1} \frac{1}{\text { in }} \mathbb{Z}$ the result will then be proved.

As in the proof of Theorem 1, it is enough to show that the 3 -decks of $A \cap \frac{1}{i n} \mathbb{Z}$ and $B \cap \frac{1}{i n} \mathbb{Z}$, restricted to multisets of form $\{0, k, \alpha\}$, are equal. Now if $a+\{0, k, \alpha\} \subset A$ then $a \in X_{A}$, and so

$$
\begin{aligned}
a+\{0, k, \alpha\} \subset A \cap \frac{1}{i n} \mathbb{Z} & \Longleftrightarrow \quad a+\alpha \in \frac{1}{i n} \mathbb{Z} \\
& \Longleftrightarrow \quad \alpha \in \frac{1}{i n} \mathbb{Z} .
\end{aligned}
$$


Thus the relevant parts of the 3-decks of $A \cap \frac{1}{i n} \mathbb{Z}$ and $B \cap \frac{1}{i n} \mathbb{Z}$ are equal, and hence $A \cap \frac{1}{i n} \mathbb{Z}=B \cap \frac{1}{i n} \mathbb{Z}$.

Theorem 8 Let $A \subset \mathbb{Q}^{n}$ be locally finite. Then $A$ is reconstructible from its 3-deck.

Proof. Similar to the proof of Theorem 7, with modifications as indicated in the proof of Theorem 6 .

Theorem 9 Let $A \subset \mathbb{R}$ be locally finite. Then $A$ is reconstructible from its 3-deck.

Proof. Let $\{q: q \in I\}$ be a Hamel basis for $\mathbb{R}$ over $\mathbb{Q}$, where the set $I$ is well-ordered by $\prec$. This induces a total ordering on $\mathbb{R}$ by defining $x<y$ iff $y-x=\sum_{i=1}^{n} a_{i} q_{i}$ with $q_{1} \prec q_{2} \prec \cdots \prec q_{n}$ and $a_{1}>0$. Given a subset $S \subset R$ we write $\langle S\rangle$ for the collection of finite $\mathbb{Q}$-linear combinations of elements of $S$.

Now suppose that $A, B \subset \mathbb{R}$ are locally finite, and that the 3 -decks of $A$ and $B$ are the same. Let $r$ be a distance that occurs in $A$ and let $X_{A}=$ $\{a \in A: a+r \in A\}$, and $X_{B}=\{b \in B: b+r \in B\}$. We may assume that $\min X_{A}=\min X_{B}=0$. Let $I_{0} \subset I$ be a finite subset of $I$ such that $x-y \in\left\langle I_{0}\right\rangle$ for all $x, y \in X_{A} \cup X_{B}$, and also $r \in\left\langle I_{0}\right\rangle$. Such a subset exists, since $X_{A} \cup X_{B}$ is finite and every element of $\mathbb{R}$ can be written as a $\mathbb{Q}$-linear combination of a finite set of elements from $I$.

We will show that for finite subsets $J$ with $I_{0} \subset J \subset I$, the sets $A \cap\langle J\rangle$ and $B \cap\langle J\rangle$ are equal, from which it easily follows that $A=B$. Consider then such a $J$. If $a+\{0, r, \alpha\} \subset A$ then $a \in X_{A}$ and

$$
\begin{aligned}
& a+\{0, r, \alpha\} \subset A \cap\langle J\rangle \quad \Longleftrightarrow \quad a+\alpha \in\langle J\rangle \\
& \Longleftrightarrow \quad \alpha \in\langle J\rangle \text {. }
\end{aligned}
$$

Since $\langle J\rangle$ is isomorphic to $\mathbb{Q}^{N}$, for some $N$, and, by the argument above, the 3-decks of $A \cap\langle J\rangle$ and $B \cap\langle J\rangle$ restricted to multisets of form $\{0, r, \alpha\}$ are the same, it follows from Theorem 8 that $A \cap\langle J\rangle=B \cap\langle J\rangle$. Since $\bigcup_{J \supset I_{0}}\langle J\rangle=\mathbb{R}$, we have that $A=B$.

It would be interesting to have a measure-theoretic version of this result. Let $S$ be a Lebesgue-measurable set of reals, and for every finite set $X$, define $S(X)=\lambda(x: X+x \subset S)$. Call $S$ locally finite if $S(X)$ is finite whenever $|X|>1$. We regard sets $X, Y$ as equivalent if $\lambda(X \triangle(Y+t))=0$ for some 
THE ELECTRONIC JOURNAL of COMBinAtorics 6 (1999), \#R20

real number $t$. Can we reconstruct every set of finite measure from its 3deck? Can we reconstruct every locally finite set from its 3-deck? Or from the $k$-deck for sufficiently large $k$ ?

\section{References}

[1] N. Alon, Y. Caro, I. Krasikov and Y. Roditty, Combinatorial reconstruction problems, J. Comb. Theory, Ser. B 47 (1989), 153-161

[2] J. A. Bondy, A graph reconstructor's manual, in Surveys in Combinatorics, 1991, ed. A.D. Keedwell, LMS Lecture Note Series 166, 221-252

[3] J. A. Bondy and R. L. Hemminger, Graph reconstruction - a survey, J. Graph Theory 1 (1977), 227-268

[4] P. J. Cameron, Stories from the age of reconstruction, Festschrift for C. St. J. A. Nash-Williams, Congr. Numer. 113 (1996), 31-41

[5] P. J. Cameron, Some open problems on permutation groups, in M. W. Liebeck and J. Saxl, eds, Groups, Combinatorics and Geometry, London Mathematical Society Lecture Notes 165, CUP, 1992, 340-351

[6] I. Krasikov and Y. Roddity, On a reconstruction problem for sequences, J. Comb. Theory, Ser. A 77 (1977), 344-348

[7] V. B. Mnukhin, The $k$-orbit reconstruction and the orbit algebra, Acta Appl. Math. 29 (1992), 83-117

[8] V. B. Mnukhin, The reconstruction of oriented necklaces, J. Combinatorics, Information and System Sciences, 20 (95), 261-272

[9] V. B. Mnukhin, The $k$-orbit reconstruction for Abelian and Hamiltonian groups, Acta Applicandae Mathematicae 52 (98), 149-162

[10] A. J. Radcliffe and A. D. Scott, Reconstruction subsets of $\mathbb{Z}_{n}$, J. Comb. Theory, Ser. A 83 (98), 169-187

[11] A. J. Radcliffe and A. D. Scott, Reconstructing subsets of non-Abelian groups, manuscript. 